\title{
CONGRES DE STRASBOURG ET NOUVELLES ORIENTATIONS DE LA SALF
}

Le 9ème congrès de la SALF s'est tenu à Strasbourg les 29 et 30 Novembre 1991. Organisé par André CLAVERT, il a connu un vif succès, avec plus de 230 participants inscrits. Deux particularités l'ont marqué :

- il s'agissait d'une réunion mixte avec la Société Allemande d'Andrologie, ce qui a permis de nombreux et fructueux échanges avec nos collègues d'outre rhin.

- toutes les communications libres se sont faites sous forme de posters et $3 \mathrm{~h}$ leur ont été spécifiquement réservées, ce qui favorise les échanges directs entre orateurs et congressistes intéressés. 51 posters ont été présentés. Beaucoup étaient excellents, particulièrement dans le domaine de la biologie de la reproduction.

Les 10 meilleurs ont fait l'objet d'une courte présentation orale, et 5 prix leur ont été attribués. Le choix tenait compte non seulement de la qualité du travail, mais également de l'âge du premier auteur, avec une priorité aux chercheurs les plus jeunes.

Le prix offert par le laboratoire Upjohn pour un travail sur l'impuissance a été attribué à F. Guilliano, de Paris, pour son travail sur l'utilisation du rat comme modèle animal d'étude de l'érection. Ont aussi été nominés $\mathrm{E}$. Wespes, de Bruxelles pour son remarquable ensemble d'études sur la biopsie du tissu caverneux dans différentes situations physiologiques et pathologiques, et F.M. Köln, de Giessen, pour son travail sur le taux du Vasoactive-Intestinal-Polypeptide dans le sang caverneux humain au cours de l'érection induite.

Les deux prix offerts par la SALF ont été attribués à K. Hinsch, également de Giessen, pour un ensemble d'études sur les protéines G de la membrane des spermatozoides, et à S. Hamamah, de Tours, pour sa contribution originale sur l'utilisation de la résonance magnétique nucléaire pour l'identification des marqueurs biochimiques du plasma séminal.

Les deux prix offerts par lintermédiaire de la Société Allemande d'Andrologie par les Laboratoires Schering et Bayorpharm ont été attribués à Mme H. Leiss, de Strasbourg, pour son étude sur l'origine du lysozyme dans le tractus génital humain, et à H. Pusch, de Graz (Autriche), pour son étude sur la fonction spermatogénétique chez les adeptes du body-building. Ont également été nominés F. Becmeur pour sa très intéressante étude sur la régulation physiologique de la température des bourses dans l'espèce humaine, ainsi que les travaux remarquables de C. Gagnon, de Montréal, sur le rôle de l'activité peroxydante des polynucléaires dans l'impact de l'infection génitale sur la fertilité masculine, et de R. Mieusset, de Toulouse, sur la réduction des taux de fécondation et de survie embryonnaire après hyperthermie transitoire des bourses chez le bélier. Cette formule poster sera développée et améliorée lors du Congrès de Nîmes, sous la responsabilité de E. WESPES.

L'Assemblée Générale a permis de présenter les nouvelles orientations de la société :

- Promotion et éventuellement sponsorisation de réunions plus spécialisées que le congrès annuel, consacrées à un sujet pointu. La réunion "Aspects récents de l'exploration et du traitement de l'impuissance" qui s'est tenue le 15 Octobre à Paris et a rencontré un très vif succès en a été le premier exemple. Nous demandons aux membres de la société de faire des propositions de thèmes, ou de soumettre des projets personnels.

- Reprise possible d'une formule "clubs d'Andrologie" consacrés à des thèmes spécialisés (voir lettre à l'éditeur de L. Rollet).

- Mise en place d'une commission d'Enseignement ayant comme mission la fédération des enseignements universitaires francophones, et à travers celle-ci l'élaboration d'un cadre commun pour l'enseignement de l'Andrologie. Cet accord général à propos de l'Andrologie, qui se concrétisera également par la participation des différents enseignements universitaires à l'élaboration de la session d'enseignement annuelle de la SALF, facilitera la reconnaissance de l'Andrologie en général, et celle de la compétence correspondante en particulier. Tous les enseignements français ont déjà donné leur accord de principe.

- Projet de constitution assez rapide d'une commission d'évaluation, destinée à mettre en place des études multicentriques visant à évaluer objectivement explorations et traitements (médicaments, PMA, chirurgie) dans le champs de l'Andrologie.

- Projet de constitution d'une commission de type éthique visant à réfléchir sur nos comportements, et peut-être à élaborer un guide de type "bonnes pratiques cliniques en Andrologie", qui pourrait également contribuer à ta reconnaissance de l'Andrologie.

Tous ceux qui sont intéressés à collaborer à l'un de ces programmes sont invités à se manifester en m'écrivant.

J. BUVAT

\section{LE MOT DU SECRETAIRE}

La Société d'Andrologie de Langue Française a pris son essor en Juillet 1982. Elle a eu 3 Présidents, J.C. Czyba, R. Schoysman et J. Buvat, et 2 secrétaires généraux puisque J.F. Guérin a tenu pendant 2 ans cette fonction que j'ai reprise en Décembre 1990. Une Société ne vit que si les membres qui régissent sa destinée ne "bénéficient" pas d'une stabilité sclérosante ; mais il faut aussi que ces membres soient motivés par le désir de dynamiser leur société. Ceux d'entre eux qui ont à cœur que la société ne piétine pas doivent donc pouvoir, sans trop de restrictions, participer aux instances dirigeantes. Les changements de statuts récemment proposés aux membres de la SALF visaient donc à trouver un juste équilibre entre ces deux exigences.

Ces modifications ont été adoptées par l'Assemblée Générale du Congrès de Strasbourg. Les membres du Conseil d'Administration seront désormais au nombre de 15. Ils seront élus pour 3 ans avec un renouvellement annuel par tiers. Leur mandat ne pourra pas excéder 6 années consécutives. Les membres du bureau seront autorisés à poursuivre leur fonction (président, secrétaire, trésorier...) pendant l'année qui suit leur sortie du CA, si leur mandat de 3 ans s'achevait avant la fin de leur deux années de fonction dans le bureau. Ainsi, une continuité sera préservée dans la direction de la SALF. L'Assemblée générale a également souhaité que les membres du CA qui seraient absents des réunions de ce conseil plus de 2 fois, sans cause valable, s'exclueraient d'eux-mêmes de la direction de la société.

Depuis sa création, la SALF a cherché à favoriser l'enseignement de l'Andrologie. Deux orientations ont donc été prises récemment :

- d'une part, les congrès de la Société seront précédés d'une journée d’enseignement qui sera chaque fois sous la responsabilité du Président du Congrès ; 
- d'autre part, un effort est entrepris pour donner une base commune aux divers enseignements d'Andrologie qui existent en France. Chacune conservera, bien entendu, sa spécificité découlant des orientations prises par les différentes équipes dans leurs travaux de recherches. Cette base commune pourrait déboucher sur la reconnaissance d'une qualification en Andrologie.

La SALF est une société vivante et le nombre sans cesse croissant de candidatures nouvelles provenant de tous les points de la Francophonie en est le témoin palpable (35 nouveaux membres en 1991). Elle est aussi une société à entrées multiples, et les thèmes qui passionnent certains sont peu appréciés par d'autres. Y. Englert a donc proposé de réduire à une fois tous les deux ans la fréquence des Congrès, tandis que des réunions plus pointues sur des thèmes précis (en relation avec l'impuissance, la FIV masculine, les effets des hormones mâles sur les métabolismes etc...) seraient organisées par des chercheurs intéressés. Cette proposition a été portée à la connaissance de l'ensemble des membres de la SALF qui, à Strasbourg, ont conclu que tant que les congrès continueront à être fréquentés de façon satisfaisante comme ils l'ont été jusqu'ici, il n'y a pas de raison valable pour les espacer.

L'Andrologie marche quelques pas derrière sa sceur la Gynécologie. En témoignent le foisonnement d'études sur les effets des hormones femelles sur les métabolismes lipidiques et la carcinogénèse, recherches soutenues par l'industrie... et la rareté de celles qui portent sur les hormones mâles.

Un des vœux du Secrétaire Général est de réussir à mettre en chantier une pareille étude chez l'homme afin, par exemple, de dissiper, ou de donner un fondement solide à la crainte du développement d'un cancer prostatique après administration d'androgènes. Quelle que soit la spécialité des membres de la SALF, ils pourront participer à cette étude. Et à d'autres travaux que les membres de la SALF, son président en tête, souhaitent initier. Si done vous avez les moyens, même modestes, de participer à la réalisation d'un de ces projets qui seront bientôt présentés, faites en part au bureau de la Société qui vous en sera très reconnaissante.

Mais comment faire en sorte que les sociétaires s'impliquent plus activement dans les activités de la SALF? Je voudrais à ce sujet raconter une petite anecdote.

J'ai rencontré un jour, à l'aéroport de OUAGADOUGOU, un jeune médecin qui m'a passionné par une vision très originale du rôle, en Afrique, des médecins originaires des pays développés. Il s'étonnait de ce que l'on insiste tant sur la quasi impossibilité de mettre en cuvre une politique de vaccination acceptée par la population autochtone. On avait pris, disait-il, l'habitude de considérer que cette population était incapable de comprendre les avantages de ces campagnes de vaccination et de se plier à la discipline nécessaire pour les rendre efficaces.

Or, ajoutait-il, personne ne prend en compte les particularités culturelles de ces populations et leurs habitudes ancestrales. Personne ne cherche à leur faire comprendre avec les mots qu'ils sont susceptibles d'intégrer facilement, ce que sont les bénéfices et les bienfaits de ces gestes tout à fait insolites pour eux. En outre, il faut imaginer ce que peuvent être les difficultés de transport dans des pays de dimensions aussi vastes. Lorsqu'au contraire, on va vers eux, comme je l'ai fait moimême, on s'aperçoit alors qu'ils sont tout à fait ouverts à ces méthodes de prévention. J'ai vécu pendant des mois dans une case dans la brousse. Je me suis marié avec une des jeunes filles du village et j'ai le sentiment d'avoir à la fois saisi les besoins profonds de ces hommes et femmes de l'Afrique profonde, et de leur avoir rendu de réels services.

Ce qui frappe le Secrétaire de la SALF, c'est la rareté des lettres adressées par ses lecteurs à leur revue. Quels que soient les sujets que nous avons abordés dans la rubrique courrier des lecteurs, quelques soient les sujets de pathologie qui ont été évoqués (sauf celui de la vasectomie), notre bulletin n'a jamais reçu qu'un flux particulièrement réduit de lettres. Il est pourtant de nombreuses difficultés thérapeutiques que vous avez rencontrées, et d'innombrables observations que vous avez faites, qui pourraient rendre service à l'ensemble de la Communauté Andrologique Francophone.

Avec quelle entité faudra-t-il donc que la SALF se marie, et dans quelle case devra-t-elle habiter, pour que "Andrologie" devienne une tribune permanente pour tous ses membres, et que ceux-ci se décident à donner une nouvelle vie à la grande famille de la SALF ? Nous sommes, pour vous y pousser, prêts à tout... ou presque !

Peut-être devons-nous penser que cette "grande" question a reçu un début de réponse au Congrès de Strasbourg ! En effet, pour la première fois, les candidatures au Conseil d'Administration de la Société ont été très nombreuses puisqu'il y avait 13 candidats pour les 5 sortants (Etcheverry, Harter, Jegou, Sele, Wespes). Et c'est donc parmi eux qu'ont été élus pour 3 ans : P. Bondil, M. Drosdowsky, R. Mieusset, J. Belaisch, J. Tignol et E. Wespes.

J. BELAISCH

\section{COURRIER DES LECTEURS : LE RETOUR DE CLUBS D'ANDROLOGIE ?}

J'aimerais une modification du fonctionnement de la SALF. Ayant été participant, pratiquement dès le début, au club d'Andrologie, j'y avais trouvé la possibilité de réaliser un véritable travail et les discussions me semblaient beaucoup plus bénéfiques que lors des "grands congrès" de la SALF.

Alors que je faisais encore de l'endocrinologie et de la diabétologie, j’avais eu l'occasion de participer à quelques groupes qui avaient mis sur pied, notamment, un matériel audiovisuel concernant le diabète. Cette expérience m'avait amené à réfléchir sur la création d'un "mini-club" d'Andrologie qui pourrait se réunir une à deux fois par an. Ces réunions seraient des réunions de travail, peut être couplées à des moments de détente et des séances "touristiques".

Ce groupe restreint pourrait travailler sur des sujets très précis et éventuellement ponctuels. Il s'agirait de faire des mises au point ou d'envisager une recherche prospective en fonction d'études bibliographiques affinées. Très récemment, j'ai eu l'occasion de rencontrer un groupe de chirurgiens pédiatriques qui organisent de telles réunions une fois par an, et cette année le thème était celui de la conduite à tenir sur la varicocèle de l'enfant. Nous avons beaucoup discuté pendant une journée et je pense que la séance a du être profitable pour tous.

J'avais envisagé plusieurs sujets :

- Réalisation d'un film (ou autre système audiovisuel) concernant le bilan de la stérilité et la prise en charge des couples,

- Recherche concernant les troubles de la spermatogénèse associées aux anomalies caryotypiques,

- Perturbation de la spermatogénèse chez les sujets porteurs de polykystose rénale,

- Protocole précis d'exploration de l'impuissance des diabétiques et surtout information au sein des associations de diabétiques,

- Protocoles thérapeutiques multicentriques.

Comme vous pourrez le constater ces thèmes sont variés et bien évidemment cette liste n'est pas limitative. Des séances de travail intensif et de "brain storming" permettraient certainement de progresser dans certains domaines. Peut être serait-il utile que nous nous réunissions pour discuter de façon plus approfondie non seulement de la liste de sujets d'intêrêts communs mais surtout l'organisation de tels groupes. 